\title{
MSC-based therapy in female pelvic floor disorders
}

\author{
Yizhen Sima and Yisong Chen ${ }^{*}$ (10)
}

\begin{abstract}
Mesenchymal stem cells (MSCs), also referred to as multipotent stromal cells or mesenchymal stromal cells, are present in multiple tissues and capable of differentiating into diverse cell lineages, holding a great promise in developing cell-based therapy for a wide range of conditions. Pelvic floor disorders (PFDs) is a common degenerative disease in women and may diminish a woman's quality of life at any age. Since the treatments for this disease are limited by the high rates of recurrence and surgical complications, seeking an ideal therapy in the restoration of pelvic floor function is an urgent issue at present. Herein, we summarize the cell sources of MSCs used for PFDs and discuss the potential mechanisms of MSCs in treating PFDs. Specifically, we also provide a comprehensive review of current preclinical and clinical trials dedicated to investigating MSC-based therapy for PFDs. The novel therapy has presented promising therapeutic effects which include relieving the symptoms of urinary or fecal incontinence, improving the biological properties of implanted meshes and promoting the injured tissue repair. Nevertheless, MSC-based therapies for PFDs are still experimental and the unstated issues on their safety and efficacy should be carefully addressed before their clinical applications.
\end{abstract}

Keywords: Mesenchymal stem cells, Mesenchymal stem cells transplantation, Cell- and tissue-based therapy, Pelvic floor disorders

\section{Background}

Pelvic floor disorders (PFDs) are a group of degenerative conditions that include urinary incontinence (UI), fecal incontinence, pelvic organ prolapse (POP), and other sensory or emptying abnormalities of the lower urinary and gastrointestinal tracts, caused by the weakening of pelvic floor supportive tissues and occurring independently or simultaneously. PFDs have an extremely high prevalence in women, affecting almost $25 \%$ of women older than 20 years in the United States, and UI is the most common disorder with a prevalence of $17 \%$ in the general population [1]. Stress urinary incontinence (SUI) is the subtype of UI, and about $50 \%$ of UI patients are classified as having SUI [2]. Despite being a common

*Correspondence: cys373900207@qq.com

Department of Gynecology, Obstetrics and Gynecology Hospital of Fudan University, 128 Shen Yang Road, Shanghai 200011, People's Republic of China disease, the exact etiology and pathogenesis of PFDs remain poorly understood. Many risk factors are related to PFDs [3], including parity, vaginal delivery, age, menopause, chronic cough, obesity, and constipation. These factors may cause abnormal metabolism of extracellular matrix and dysfunction of the pelvic supportive tissues such as cardinal and uterosacral ligaments [4], levator ani muscle [5], and urethral sphincter [6], contributing to the development of PFDs. Although the treatment principles for PFDs vary from different manifestations of the patients, current managements for PFDs can be generally divided into surgical and non-surgical treatments. Nonsurgical treatments, including pelvic floor muscle physiotherapy, biofeedback, pessaries, and electrical stimulation [7], have the effects on relieving symptoms and are recommended for the newly diagnosed patients, but they do not offer an anatomy restoration of the pelvic floor. Surgical treatments are recommended for patients who have 
failed the conservative managements, but surgical treatments are associated with notable complications $[8,9]$. The limitations of current managements for PFDs highlight the need to develop new treatments. Restoration of the pelvic floor structures and improvement of the pelvic floor functions through cell therapy has been investigated in many studies. MSCs, as a highly investigated population in regenerative medicine, hold a great promise to enhance tissue repair and have yielded therapeutic effects in a large spectrum of diseases such as graft-versus-host disease (GVHD) [10], cardiac diseases [11] and multiple sclerosis [12]. There is a growing body of literature that recognizes the effectiveness of MSCs-based therapy for treating PFDs both in preclinical experiments and in a small number of clinical trials. Although most of them are preliminary studies, the symptoms of PFDs were relieved both in animal models and human subjects.

This review summarizes the cell sources of MSCs used for PFDs and the roles MSCs playing in the treatment of PFDs, and analyses the recent studies concerning MSCbased therapy for PFDs. It is hoped that this review will contribute to a better understanding of MSCs applications in disease therapy and provide references for the future investigations on MSC-based therapy in PFDs.

\section{MSCs and MSC-based therapy}

Before being named as mesenchymal stem cells, MSCs were first identified from bone marrow and described as colony-forming units-fibroblasts for their fibroblastlike appearance and the ability to form colonies in vitro [13]. In 1990s, Caplan first put forward the term "mesenchymal stem cells" and Friedenstein et al. described the multilineage potential of MSCs that could differentiate into tissues of mesodermal origin such as adipocytes, chondroblasts, and osteoblasts in vitro [14], stimulating the imagination of this intriguing cell type in tissue regeneration. Although MSCs were first isolated from the bone marrow, they have been harvested from many other fetal and adult tissues, including adipose [15], umbilical cord [16], placenta [17], amniotic fluid [18], skin [19], and dental pulp [20]. In 2006, the International Society for Cellular Therapy established minimal criteria to define MSCs derived from multiple tissues and organs, which include the adherent plastic property, surface markers and in vitro multilineage differentiation potential of MSCs [21]. According to that criteria, many preclinical and clinical trials identified MSCs from different origins and applied MSCs into therapeutics. The biological properties such as multilineage potential and immune modulation make MSCs a promising treatment option for a variety of clinical conditions. For example, the phase III trials have been conducted in congestive heart failure [22] and Crohn's disease [23]. Furthermore, MSCs have secured conditional approval to treat children with GVHD in several countries [24].

Nevertheless, MSC-based therapies have demonstrated excellent therapeutic effects, but there are many unknowns and controversies of MSCs as well as MSCbased therapies. A particular challenge for the field is to set criteria for MSCs. With no specific marker to define MSCs, the surface markers vary between the MSCs derived from different origins [25]. Moreover, the studies identifying and characterizing MSCs are mostly based upon in vitro work, and thus it is hard to qualify the in vivo multilineage potential of MSCs.

Over the last decade, researchers have never stopped to discover the nature of MSCs. Studies revealed that MSCs derived from different origins would exhibit widely different transcriptomic signatures, biological functions, and in vivo developmental potentials [26, 27], which adds new complexities to the identification of MSCs. Besides, there is a growing body of literature recognizes that most MSCs were derived from pericytes $[28,29]$ and they functionally improved tissue repair or modulated immunity through the paracrine effect rather than differentiation [30-32]. Considering the above evidences challenging the term "mesenchymal stem cells", and to prevent the abuse of MSCs as a cure-all in business activities, academics and experts recommended to change the term into "medical signaling cells", reserving the name "MSCs" [33, 34]. Taken together, it is important to further investigate the properties of MSCs as well as to identify the criticisms of their therapeutic uses, because only then can promote their translation from the bench to the bedside.

\section{Sources of MSCs in treatment of PFDs (Fig. 1)}

Bone marrow-derived MSCs (BM-MSCs) are the first discovered MSCs, with the well-studied biological properties, usually considered as the gold standard cell type when investigating the properties of MSCs from other tissues. The previous studies reported BM-MSCs and Adipose-derived MSCs represented the optimal MSCs sources due to their outstanding differentiation capacity $[35,36]$. Also, given the immunomodulatory property, BM-MSCs have been used in the treatment of GVHD $[10,37]$. However, MSCs are relatively rare in bone marrow (approximately 1 per 10,000 cells) and traditional bone marrow procurement is painful for patients, which may restrict the application of this cell population in PFDs.

Adipose-derived stem cells (ADSCs) were first reported being used in an animal model for treating PFDs in 2010 [38]. Then a considerable number of studies focusing on ADSCs-based therapy for PFDs have been carried out both in preclinical and clinical trials. The popularity of 


\section{MSCs sources}

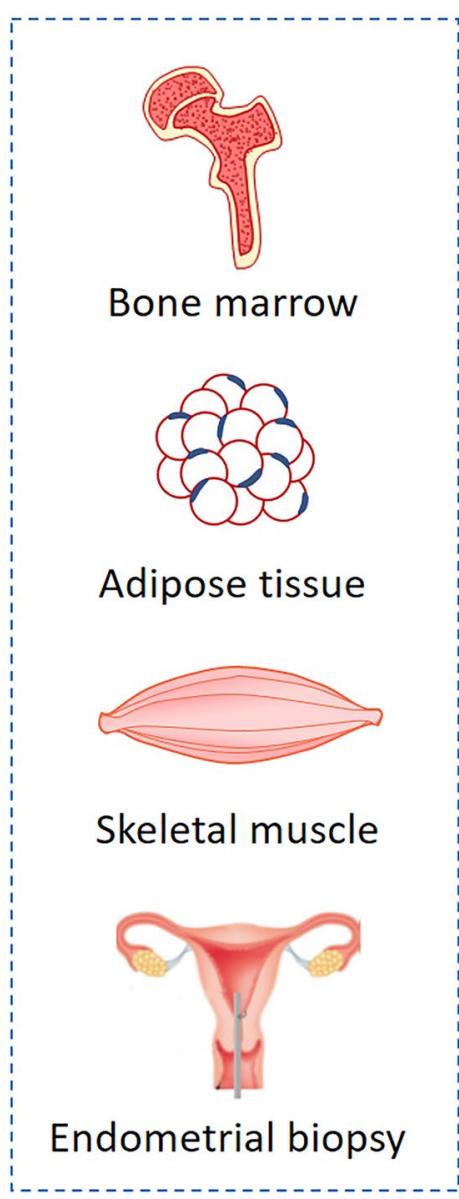

\section{MSC-based therapies}

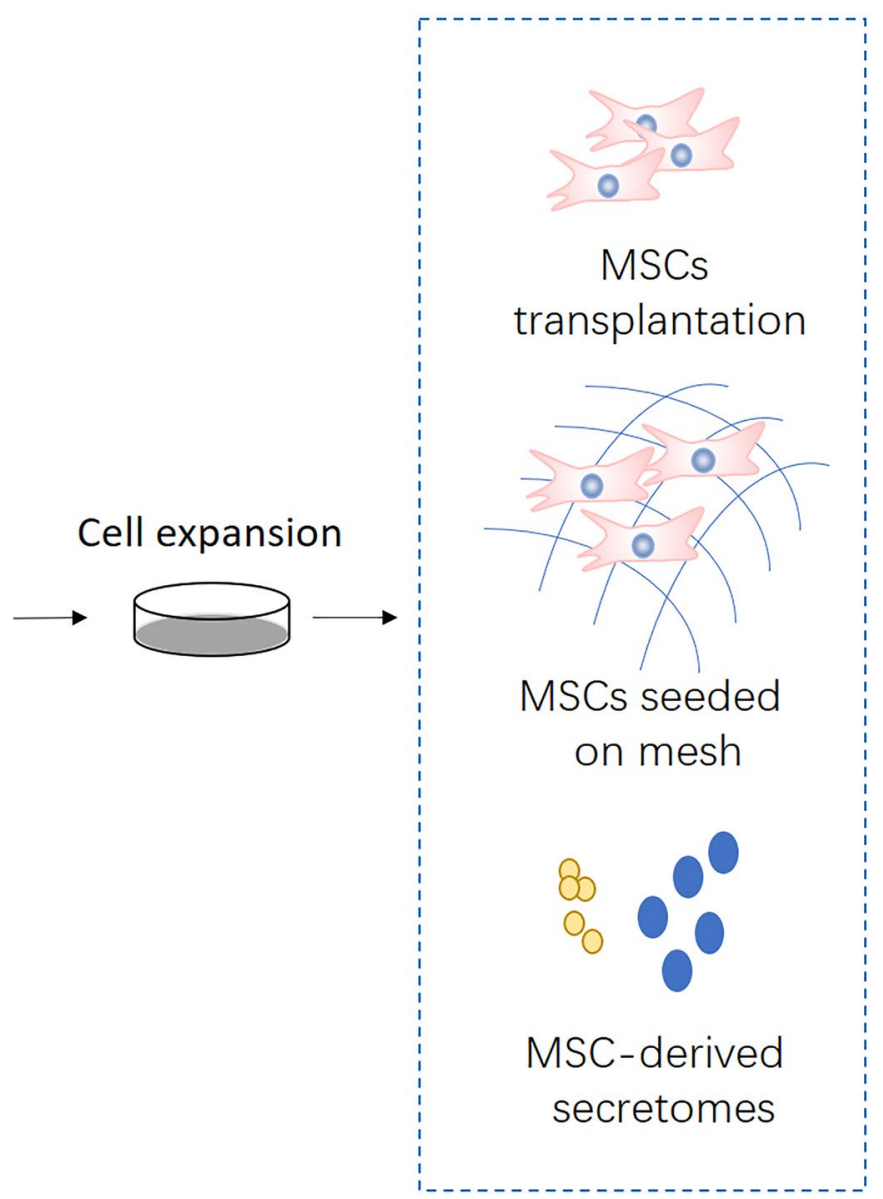

Fig. 1 Schematic representation of the different MSCs sources and MSC-based therapies for PFDs

ADSCs can be attributed to their biological properties as well as the convenient procurement. According to a clinical study utilizing ADSCs translation to treat SUI, the autologous tissue source was harvested from the patients' abdomen by liposuction; then ADSCs were isolated from the tissue without culture using the Celution system and finally injected into the urethral sphincter [39]. This treatment can be completed as a single surgical procedure within $3 \mathrm{~h}$. Besides, the injury of isolation site for ADSCs is minimal, and ADSCs are in large quantities in human adipose tissue. Due to these beneficial features for their clinical applications, ADSCs have become a highly investigated cell population in the treatment of PFDs.

Muscle-derived stem cells (MDSCs) are a population of muscle-resident stem/progenitor cells acquired through muscle tissue biopsies. In fact, there are heterogeneous populations of muscle-resident stem/progenitor cells in skeletal muscle. Apart from the well-known satellite cells that are capable of regenerating muscle fibers, there are groups of non-satellite cells with multilineage potential that are considered belonging to MSCs, which was confirmed by the wide gene expression similar to MSCs [40, 41]. MDSCs have been shown effective and well-tolerated in most clinical studies by urethral injection to treat SUI. MDSCs are also candidate cells of tissue engineering for the therapy of POP, because they are capable of promoting vaginal repair with tissue-engineered scaffolds in rat models [42]. However, the invasive acquisition procedure that often causes significant pain and morbidity is an issue to be solved for the application of MDSCs.

Endometrial MSCs (eMSCs) were isolated from human endometrium which is a highly regenerative tissue undergoing more than 400 cycles of growth and shedding during a woman's reproductive years. Adult human endometrium contains a small quantity of epithelial progenitors and MSCs, which may provide a readily available source of MSCs for cell-based therapies [43, 44]. Recently, several studies combining 
eMSCs with new biomaterials gained good results in skin wound repair or abdominal hernia animal models $[45,46]$, demonstrating eMSCs are candidate seeding cells for tissue engineered meshes in the treatment of POMoreover, the convenience of eMSCs acquisition (endometrial biopsies in an office-based procedure) and the discovery that eMSCs can be also isolated from post-menopausal endometrium [47] contribute to their potential clinical use for PFDs.

\section{More other sources}

In additional to the above MSCs, there are some other MSCs sources regarded as candidates for the therapy of PFDs with rare investigations. (1) Umbilical cordderived MSCs (UC-MSCs) are MSCs derived from various parts of the umbilical cord and particularly from Wharton's Jelly. Wharton's Jelly matrix is located close to the vasculature of the cord. MSCs derived from Wharton's Jelly are called human umbilical cord perivascular cells (HUCPVCs). The similar characteristics between HUCPVCs and BM-MSCs support the applicability of HUCPVCs for cell-based therapies [48]. It has previously been observed that UC-MSCs contributed to the repair of vaginal wall in rats by fabricating a cell-seeded tissue engineering production [49]. (2) Umbilical cord bloodderived MSCs (UCB-MSCs), extracted from human cord blood without invasive procedures, are expected to be useful for cell therapy in regenerative medicine. But the investigation of this cell source in PFDs is rare. The only clinical trial [50] suggested that UCB-MSCs transurethral injection were effective in relieving the symptoms of SUI, which were evaluated by urodynamic study. (3) Placenta-derived MSCs have attracted attention for their immune-modulatory properties and poor immunogenicity, which makes them suitable for allogeneic transplantation. Decidua-derived MSCs, derived from human term decidua, are capable of multilineage differentiation into all three embryonic layers, and they were regarded as a potential source of MSCs for PFDs [51]. (4) Urinary-derived stem cells (USCs) are a subpopulation of cells isolated from human urine, possessing MSC-like features such as clonogenicity, self-renewal, and multipotent differentiation capacity [52, 53]. Moreover, USCs can be obtained noninvasively from human urine specimens. Thus, they are thought to have potential use in genitourinary reconstruction.

MSCs isolated from different tissues exhibit important differences in their availability, characteristics, and regenerative potential. Therefore, the choice of cell source, subsequent isolation, and manipulation techniques depend on the requirements of specific research/clinical applications.

\section{Roles of MSCs in treatment of PFDs Migration to the site of injury}

MSCs have been demonstrated to migrate and situate at the site of injury following infusion, which is also termed as "homing". MSCs homing is defined as the arrest of MSCs within the vasculature of a tissue followed by transmigration across the endothelium [54]. However, it is unclear if MSCs actively home to tissues using leukocyte-like cell adhesion and transmigration mechanisms or are passively entrapped in small-diameter blood vessels [54]. Although MSCs express many receptors and cell adhesion molecules such as chemokine receptor [55] and integrins [56], the exact mechanisms underlying the migration and homing are not well understood.

Current MSC-based therapy for PFDs usually delivers MSCs by periurethral injection. But no matter what injection methods are used, it is impossible to deliver MSCs to the specific site of injury. Considering the connective tissue damages of the pelvic floor are extensive, the homing property of MSCs would play a pivotal role in treating PFDs (Fig. 2a). Cruz et al. found that intravenously injected MSCs distributed to pelvic organs after simulated childbirth injury in a rat model, suggesting that intravenous administration of MSCs could provide an effective route for cell-based therapy [57]. Ben et al. transplanted MSCs systemically or locally to vaginal injury rat model to examine the engraftment, survival, differentiation and angiogenic effect of transplanted MSCs. As a result, both systemic and local MSCs transplantation promoted host angiogenesis, while engraftment after local transplantation was less efficient at all-time points compared to systemic administration, indicating that systemically transplanted MSCs promote tissue repair through homing to the site of injury [58].

\section{Paracrine effects}

The mechanisms of MSCs therapeutic effects were initially thought as differentiation and cell replacement. However, more and more studies reveal that MSCs exist in vivo for a short time after transplant, in contrast to their long-lasting therapeutic effects. In addition, it is rare to see the injected MSCs engraft into target tissues and differentiate into desired cells. Therefore, many believe that the observed therapeutic effect of MSCs is due to their paracrine effects, also termed as hit-andrun mechanism, which are based on the production of exosomes or secretion of trophic and immunomodulatory factors during the initial days following MSC injection [59] [60]. MSCs secrete a range of proteins/ peptides, RNA, hormones, and chemicals by extracellular vesicles such as exosomes or microvesicles [61], and MSC secretions have gained remarkable therapeutic 


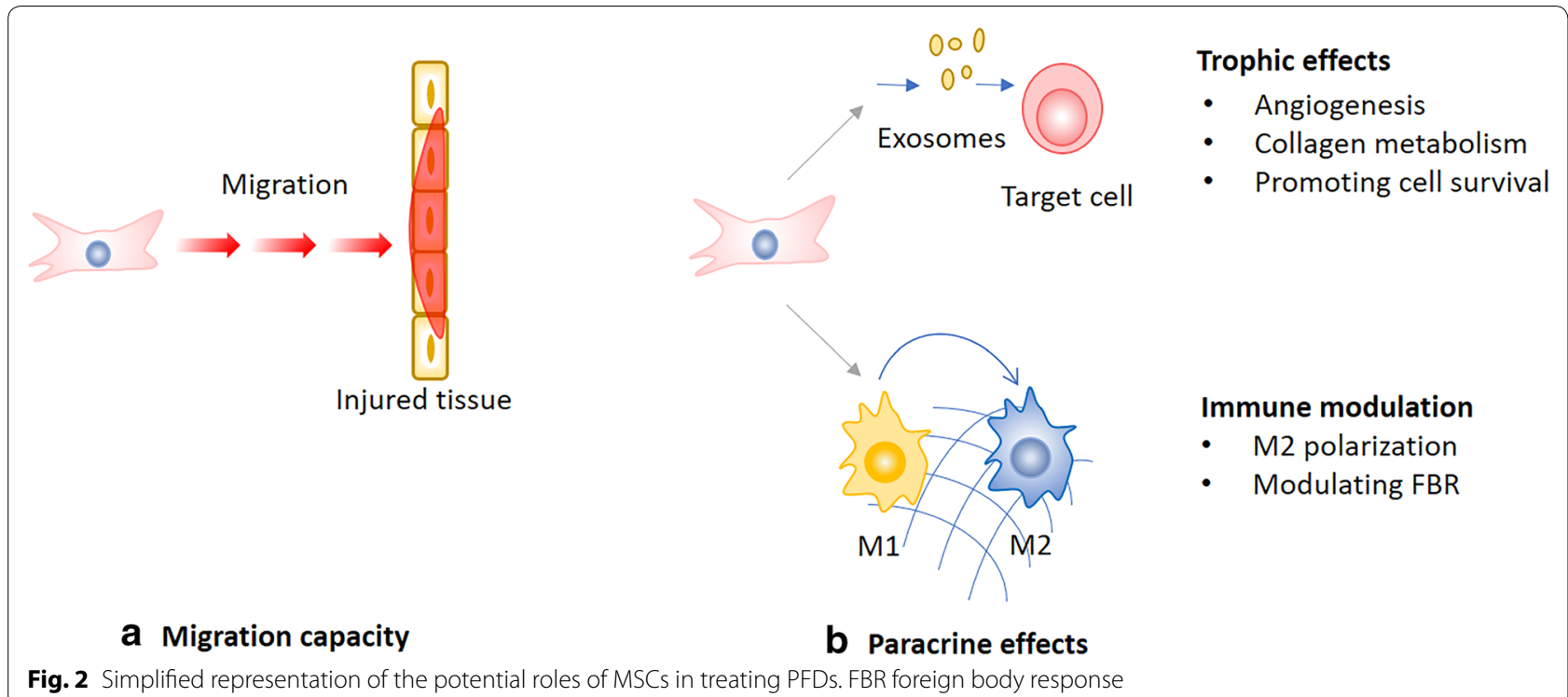

outcomes in preclinical studies. Hence, acellular therapy which harnesses MSC secretions to promote tissue repair is increasingly attractive in regenerative medicine [62]. The paracrine effects of MSCs in the treatment of PFDs can be concluded to trophic effects and immune modulation (Fig. 2b).

Promotion of cell survival or trophic effects MSCs can secrete a broad array of factors that support cell survival, including growth factors, cytokines, and extracellular matrix. Collectively, these secretions have the theoretical capacity to rescue injured cells, reduce tissue damage, and accelerate tissue repair. And this property is exemplified by the natural roles of MSCs as reticular cells that support the hematopoietic stem cell niche and as vascular pericytes that support endothelial cells [63].

In MSC-based therapy for PFDs, paracrine effects of MSCs play an important role in tissue regeneration through promoting the muscular cell survival [64], enhancing the host angiogenesis [58] and modulating the collagen metabolism [65]. Collagen metabolism disorder in connective tissues is one of the well-recognized pathogenic mechanisms of POP, and MSCs may regulate collagen metabolism via paracrine effect to optimize the functional characteristics of fibroblasts. In a preclinical study, the increased collagen I and III productions were observed after a systemic administration of exosomes in the early stage of wound healing, while in the late stage, exosomes might inhibit collagen expression to reduce scar formation [66]. On the contrary, as a novel treatment for fibrotic diseases, MSCs act to reduce TGF $\beta$-induced myofibroblasts differentiation and collagen deposition during organ fibrosis $[67,68]$.
Immune modulation There are a number of publications focusing on the pleotropic effects of MSCs on the immune system. Early studies suggested that MSCs might be immune privileged because MSCs failed to elicit alloreactive lymphocyte proliferative responses $[69,70]$. But immune rejections were reported in allogeneic MSCs transplantations. A review by Ankrum et al. [71] provides a thorough discussion on immunogenicity of MSCs and suggests that "MSCs are immune evasive and not immune privileged." The immunogenic and immunosuppressive properties of MSCs are strongly dependent on context and induced by the inflammatory factors which MSCs are exposed to. Given the diverse immunomodulatory properties of MSCs, MSC-based therapies have been applied in GVHD, sepsis, and some autoimmune diseases. Specifically, MSCs act on both the adaptive and innate immune systems by reducing the activation and proliferation of $\mathrm{T}$ and B lymphocytes, suppressing dendritic cell maturation, inhibiting proliferation and cytotoxicity of Natural killer cells, promoting M2 macrophage polarization, and increasing the number of regulatory $\mathrm{T}$ cells $[72,73]$. The mechanisms of MSCs in mediating these processes are based on their paracrine effects, by secreting inflammatory cytokines such as IL-6, IL-10, TGF-b, PGE2 [74-76].

Herein, the immunomodulatory property of MSCs plays a role in regulating the foreign body response (FBR) when treating POP with the combination of MSCs and biomaterials. FBR is the end-stage response of inflammatory and wound healing processes following medical implantation [77], and ultimately determines rejection or integration of the implanted biomaterial. Synthetic polypropylene meshes, used in pelvic floor reconstructive surgery for POP, have long been blamed for triggering 
excessive FBR and then causing mesh exposure or erosion. But now, MSCs have been proved to suppress FBR and improve the biocompatibility of meshes in animal models [78, 79] based on the cross-talk between MSCs and immune cells, particularly the macrophages. Therefore, the immunomodulatory property of MSCs may contribute to reducing the surgical complications of POP.

\section{Preclinical studies of MSCs-based therapy for PFDs}

MSCs are highly investigated as a novel therapy for PFDs in a variety of preclinical studies, with different animal models, cell sources, delivery methods and response evaluation systems. MSCs transplantation is the most extensively studied therapeutic strategy in this field, with a long history of therapeutic research for SUI. As the regenerative medicine advances, MSCs-based tissue engineering and MSC-derived exosomes or other secretions emerged as new options for PFDs (Fig. 1).

It is now well established from preclinical studies, that MSCs transplantation is a potential therapeutic strategy for urinary and fecal incontinence (Table 1).

Lin et al. [38] first published an investigation applying autologous ADSCs to treat SUI in a rat model. Rats were induced to create an abnormal voiding condition by postpartum vaginal balloon dilation and bilateral ovariectomy. ADSCs were isolated from the rat peri-ovary fat, which was different from the clinical use of the subcutaneous fat because the bilateral ovariectomy was designed to simulate menopause in rats. Then ADSCs were transplanted into the rats via urethral injection or intravenous injection through tail vein. Four weeks later, urinary voiding function was assessed by conscious cystometry and $80 \%$ of the control rats had voiding dysfunction, whereas only $33 \%$ of the ADSC-treated rats had voiding dysfunction. Normal voiding rats from the ADSC-treated group had significantly higher smooth muscle content and elastin content than the control group or ADSC-treated abnormal voiding rats. These findings suggested that transplanted ADSCs could improve urethral function and the migration of ADSCs toward the injured urethra might be one of the steps through which voiding dysfunction was mitigated. Notably, in this early time research, the labeled MSCs were detectable in the connective tissue till 4 weeks post-transplantation. However, later studies reported that MSCs survived for a short time in spite of producing long-term tissue regenerating effects.

A number of research evidence on the therapeutic effects of MSCs in urinary incontinence support MSCs transplantation as a novel treatment, with restorations in urinary function and structures. However, how long MSCs can survive in situ after an injection has been a debatable question, and different studies reported different survival times of MSCs.
Cruz et al. [57] reported a pelvic organ distribution of MSCs after intravenous injection in the rats with vaginal distention (VD). MSCs were transfected and constitutively express a green fluorescent protein (GFP) which could be assessed by fluorescent Imaging. In vivo imaging demonstrated evidence of GFP + MSCs in the pelvic region both 4 and 10 days after $\mathrm{VD}$, but the total flux decreased from 4 to 10 days. Another research that evaluated the potential role of human MSCs in the improvement of urinary continence also explored the fate of injected MSCs [80]. To detect the transplanted MSCs, several approaches were conducted: nuclei were traced using in situ hybridization for human Alu genomic repeats via digoxigenin-labeled DNA probes; in vivo bioluminescence imaging (BLI) was applied to assess MSCs viability and distribution after local periurethral injection in real time. The results showed positively Alu-stained nuclei were observed at $2 \mathrm{~h}$ after injection, but were not observed 4,10, and 14 days after locally and systemically MSCs transplantation. PKH26-labelled cells were also found at 2 and $24 \mathrm{~h}$ after injection. BLI signals increased 1 and 2 days after MSCs injection in VD rats, while no significant difference was observed in non-VD rats. (Figure 3). These findings suggested intravenously injected MSCs migrated to the site of injury, which provided an effective route for cell-based therapy to treat SUI; furthermore, MSCs did not promote tissue regeneration in the way of differentiation and replacement considering their rapid disappearance.

Recently, Menachem-Zidon et al. [58] reported a study evaluated the survival, differentiation and angiogenic effects of transplanted MSCs in a vaginal injury rat model established by vaginal incision. Remarkably, the systemically transplanted cells labeled with green florescent protein (GFP) migrated to vaginal injury site and survived for at least 30 days; furthermore, the transplanted cells acquired an endothelial phenotype in vivo, and they were detectable within capillary-like structures. By ruling out the occurrence of fusion between transplanted MSCs and the host endothelial cells, the authors proposed the transplanted MSCs differentiated in situ into endothelial cells. However, the authors didn't explain why the long-term existence, as well as the endothelial phenotype were only observed in rats with systemically transplanted MSCs, not in those with locally transplanted MSCs. As a matter of fact, whether MSCs are capable of infusion and differentiating into tissue cells, are still controversial. More studies need to be conducted to investigate the fate of transplanted MSCs in vivo.

Apart from the investigations on urinary incontinence, MSCs transplantation has also been studied for fecal incontinence (FI). Salcedo et al. [81] reported that MSCs improved the anal sphincter function in rats with 


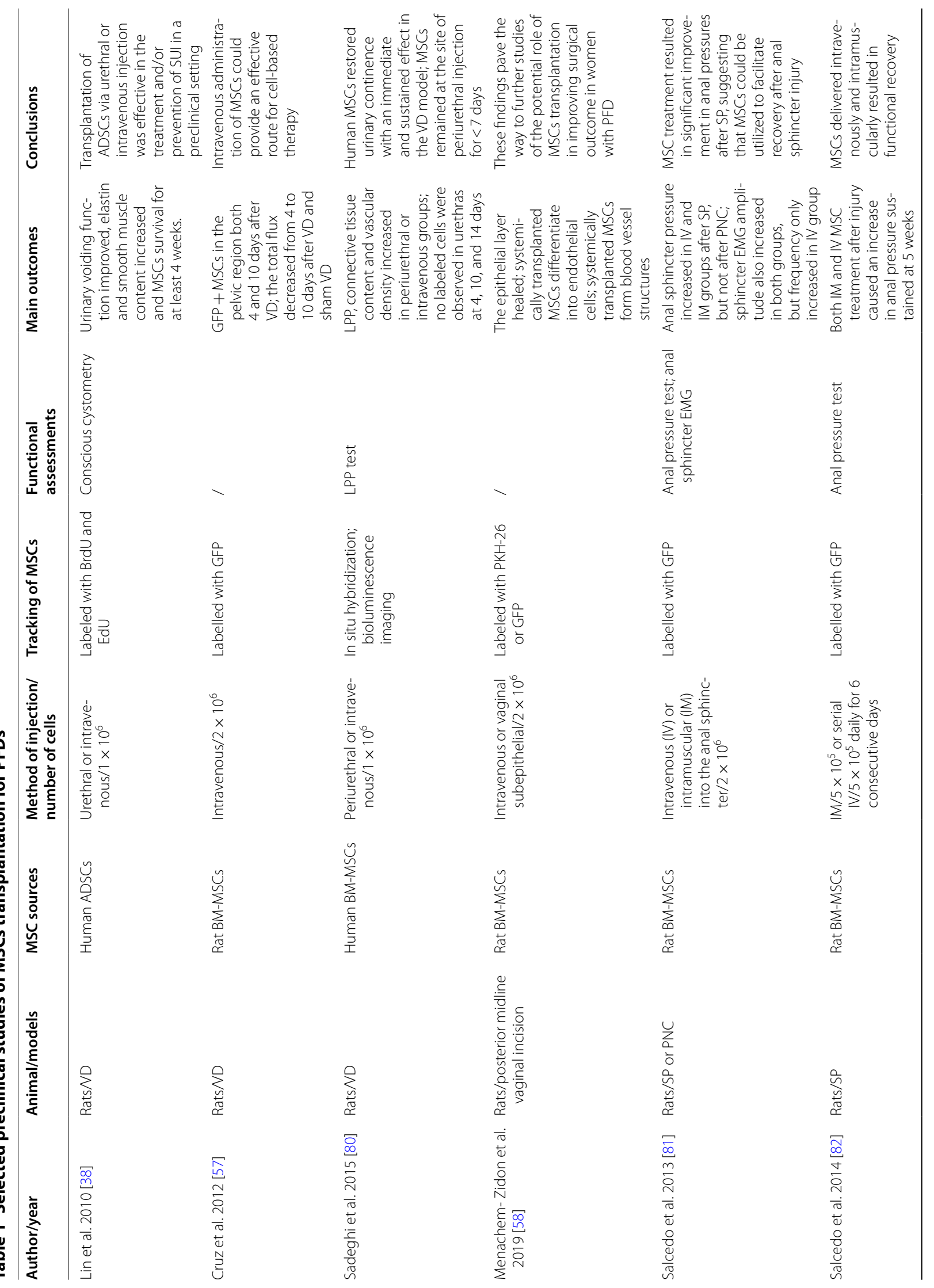




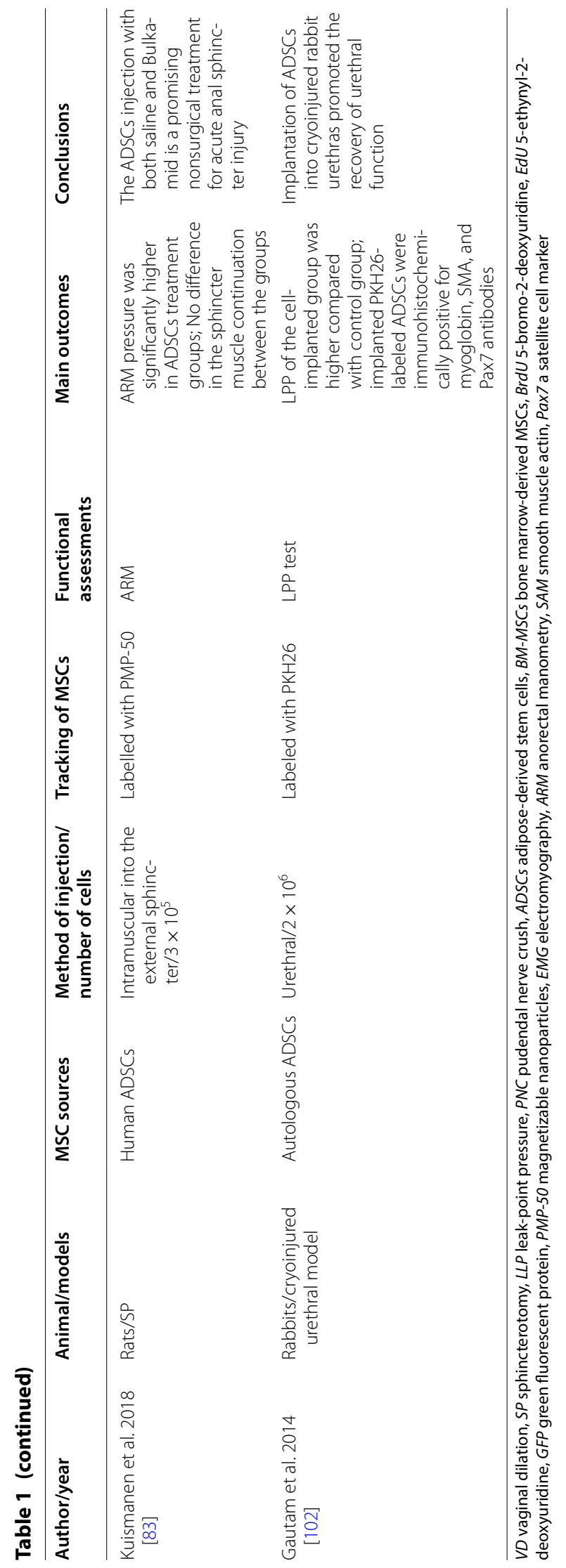




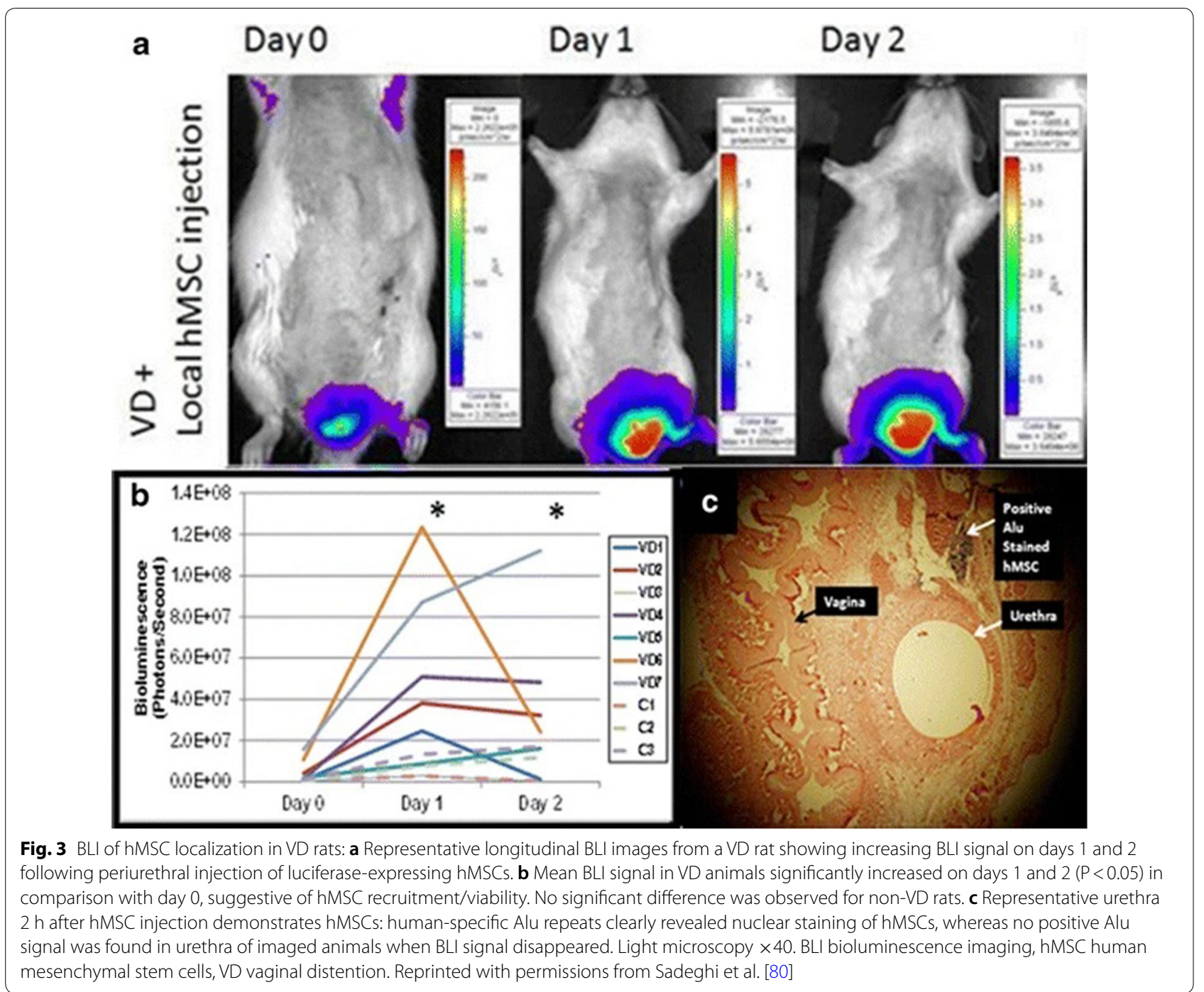

anal incontinence caused by sphincterotomy, but they only measured the anal sphincter pressure 10 days after injury. After that, they further investigated the regenerative effects of MSCs on the injured anal sphincter by comparing anal sphincter pressures following intramuscular and serial intravascular MSCs injection [82]. Anal sphincter pressure increased in both intramuscular and intravascular injection groups, and the increase lasted for 5 weeks. Also, in both MSCs treated groups, less fibrosis and more collagen deposition were found, with the intravascular injection group showing the least scarring. Kuismanen et al. [83] reported similar results of an increase in anal sphincter pressure after MSCs delivery in the same animal model. In addition, a biocompatible carrier, polyacrylamide hydrogel Bulkamid was found to be a suitable carrier for MSCs, because Bulkamid well integrated into the tissue, and a minor foreign body reaction was found in the group receiving Bulkamid with MSCs.

In addition to the efficacy of MSCs treatment, safety of the therapy is a matter of concern. Despite the well-tolerated outcomes of transplanted MSCs in most rat models, there remains a paucity of evidence on the long-term, comprehensive evaluation of the safety. A study tested the dose-effect safety profile of skeletal muscle precursor cells therapy in a sphincter-removed dog model [84]. No adverse effects were found according to the histological pathologic features, blood cell counts, or liver and kidney function markers up to 9 months after cell injection (25100 million cells per milliliter). This is probably equivalent to around 2-3 years of follow-up in humans. 
Sima and Chen Cell Biosci $\quad$ (2020) 10:104

Page 10 of 21

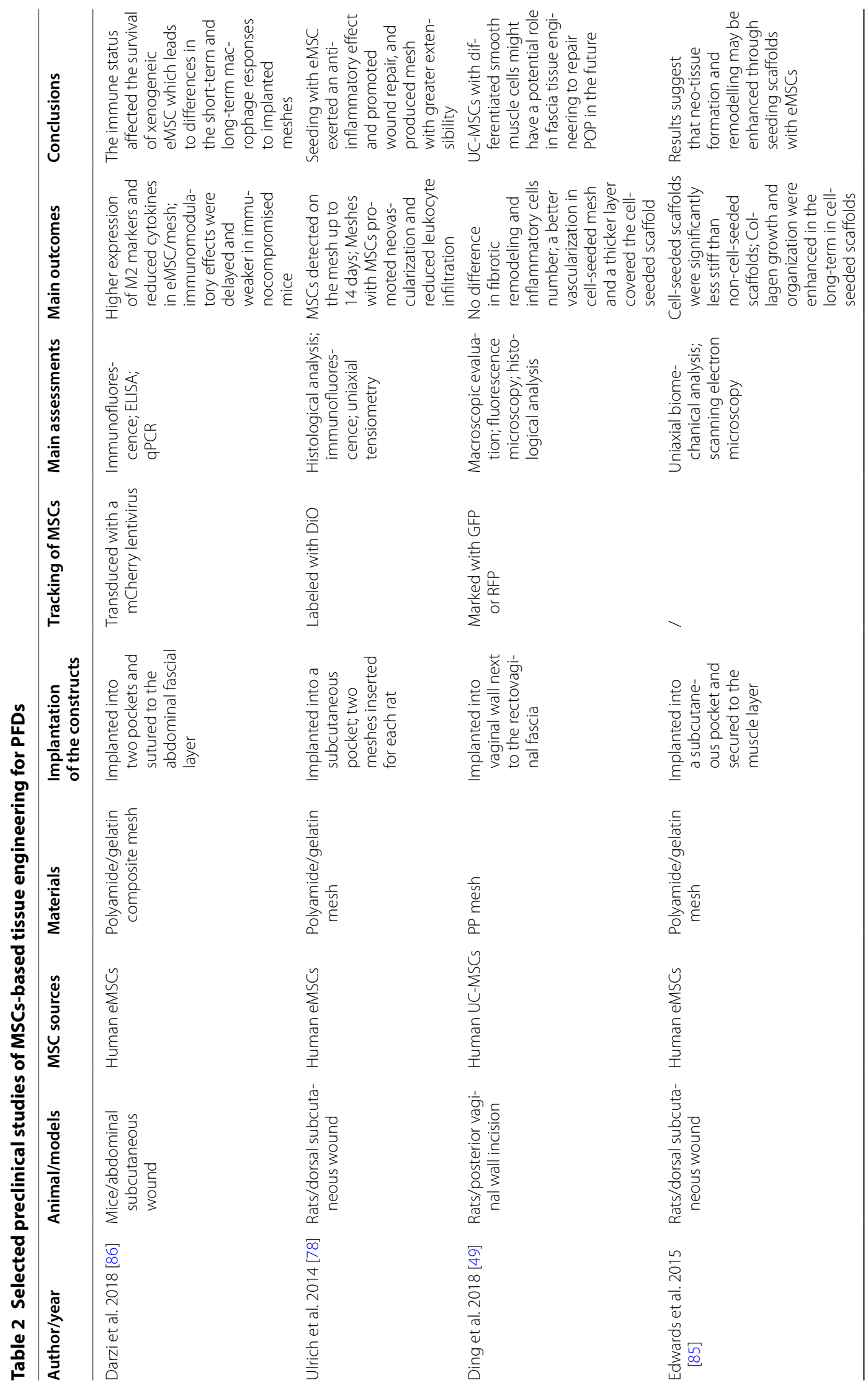




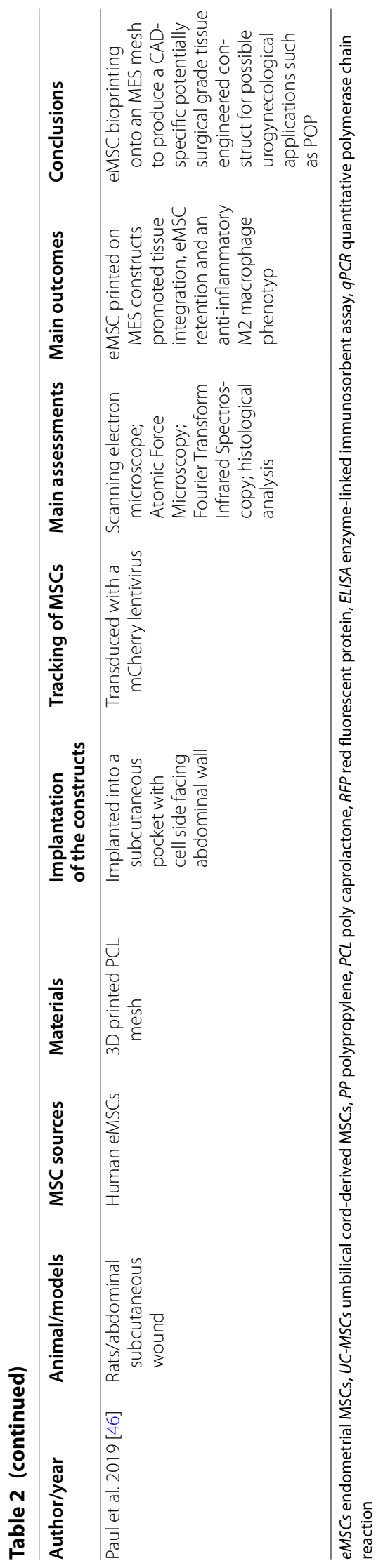




\section{MSCs-based tissue engineering for PFDs}

Therapies involving tissue engineering, combining MSCs with new materials or meshes were also evaluated in several studies, particularly in the treatment of POP (Table 2). POP is the herniation of pelvic organs into the vagina; hence, the meshes implantation can compensate for inadequate or lack of autologous tissues, to decrease morbidity and to improve long-term efficacy. Many reported MSCs and meshes assisted each other and interacted to improve the final outcomes as a combination. Meshes provide mechanical and structural support for the pelvic tissues as well as offer the cells a scaffold to adhere. Meanwhile, MSCs exhibit immunomodulatory and anti-inflammatory properties to suppress the excessive FBR. Thus, tissue engineering is a new option in the field of pelvic floor repair when soft tissue reinforcement is necessary.

In recent years, there has been an increasing interest in applying eMSCs to tissue engineering therapy for pelvic floor repair $[46,78,85]$ and the eMSCs have exhibited an excellent modulatory property to the extra cellular matrix remodeling and the inflammatory reactions, but the mechanism remains unclear. A study [86] characterized some of the immunomodulatory properties of eMSCs in vivo to understand the immunoregulatory mechanism of eMSCs on macrophages. The authors implanted polyamide/gelatin composite mesh seeding with mCherry lentivirus-labelled eMSCs to the abdominal subcutaneous wounds in C57BL6 immunocompetent and NSG immunocompromised mice. Dual color immunofluorescence staining was performed to quantify M1 and M2 macrophages. Results showed that eMSCs were detected around the mesh in NSG mice but not in C57BL6 mice on 3 and 7 days after implantation (Fig. 4). Both in NSG and C57BL6 mice models, the $\mathrm{M} 2 / \mathrm{M} 1$ ratio was higher and the expression of M2 macrophage markers increased in eMSC/mesh compared to mesh control. Also, the inflammatory cytokine IL-1 $\beta$ and TNF- $\alpha$ reduced in eMSC/mesh compared to mesh control. These immunomodulatory effects were delayed and weaker in NSG mice compared to C57BL6 mice. In sum, the eMSC-modulated macrophage responses to synthetic meshes differed in immunocompetent and immunocompromised mice. It is apparent that the eMSC exerted these immunomodulatory effects via a paracrine mechanism, since eMSCs disappeared rapidly after implantation while the anti-inflammatory effect lasted to 30 days.

\section{MSCs-derived secretome for PFDs}

The spectrum of regulatory and trophic factors secreted by MSCs, including exosomes, cytokines, and chemokines, is broadly defined as the MSC secretome. With an awareness shift of MSCs therapeutic effects from differentiation to secretion, many studies harnessed MSC secretome to treat various diseases [30, 87]. This new MSCs-based therapy, known as acellular therapy, can provide therapeutic benefits without the need to transplant living cells, making the process easier to be standardized and reducing cell transplant related risks. Several attempts have been made to investigate the therapeutic effects of MSC secretome in PFDs (Table 3), by utilizing the concentrated conditioned media (CCM) of MSCs $[64,88]$ or the exosomes derived from MSCs [89] [90].

An in vivo study from Liu et al. [65] evaluated the effects of human MSC-derived exosomes on collagen metabolism in cultured fibroblasts from postmenopausal women with or without SUI. Exosomes were prepared by ultracentrifugation of MSCs conditioned medium and were confirmed by transmission electron microscopy and western blot analysis. After 6-h culture, the expression of type I collagen, TIMP-1 and TIMP-3 increased, whereas the expression of MMP-1 and MMP-2 decreased in vaginal fibroblasts treated with exosomes. The results suggested that exosomes increased type I collagen contents by increasing collagen synthesis and decreasing collagen degradation in vaginal fibroblasts from women with SUI.

In another research from $\mathrm{Ni}$ et al. [89], the therapeutic potential of human ADSCs-derived exosomes in SUI was studied in vivo and in vitro. The methods of exosomes isolation and characterization were similar to Liu's research. SUI model was established by pudendal nerve transection (PNT) and vaginal dilation (VD) in female rats and rats were peripheral urethral injected with ADSCs or ADSCs-derived exosomes. In-vitro results showed exosomes could enhance the growth of skeletal muscle and Schwann cell lines in a dose dependent manner. In-vivo experiments illustrated that rats of the exosome group had higher bladder capacity and leak point pressure (LPP), and had more striated muscle fibers and peripheral nerve fibers in the urethra than rats of control grouTo explain how the exosomes benefit the recovery of SUI, the authors performed proteomic analysis and found ADSC-derived exosomes contained a variety of proteins related to skeletal muscle and nerve regeneration, but the precise mechanisms underlying the phenomenon are still unknown.

Similarly, a recent study [90] reported the therapeutic effects of MSCs-derived exosomes on SUI in a rat model, and illustrated the therapeutic effects in skeletal muscle regeneration were related to the phosphorylation of extracellular-regulated protein kinases (ERK) in satellite cells (SCs). Histological analysis showed fibrosis and muscle morphology were close to normal in pubococcygeus muscle after 8 weeks of exosomes injection. 
a

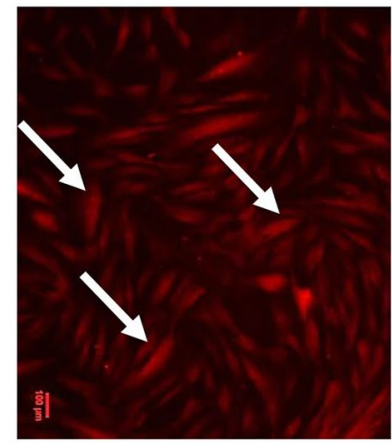

d

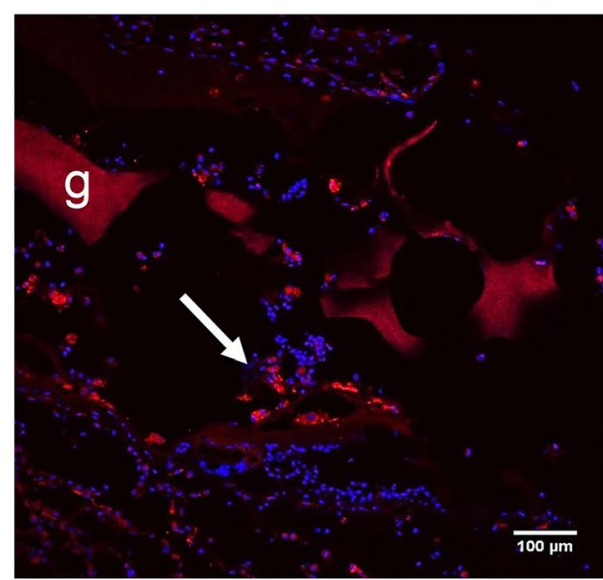

$\mathbf{f}$

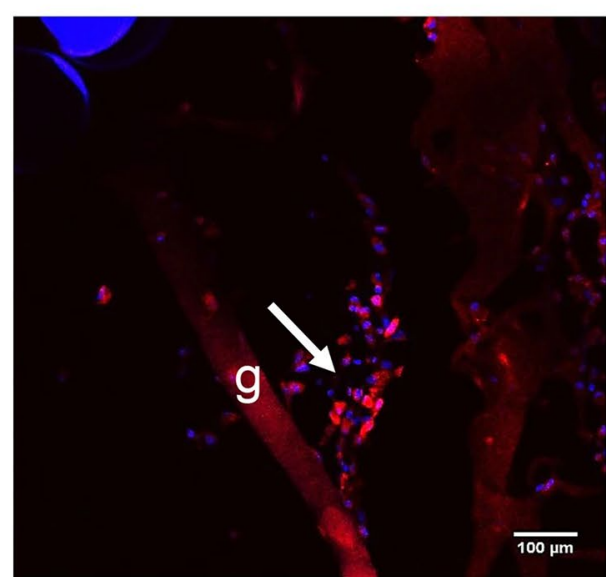

b

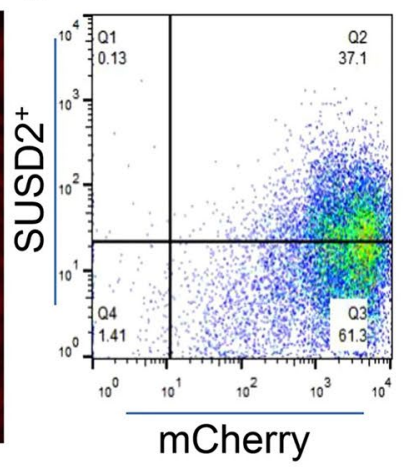

C

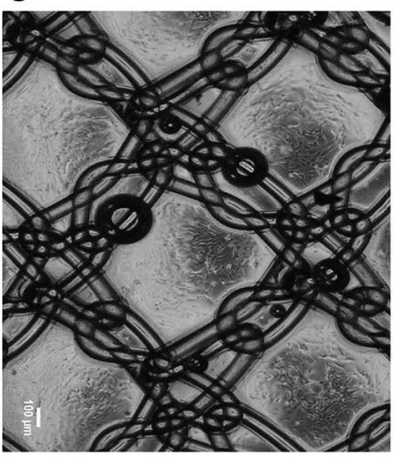

e

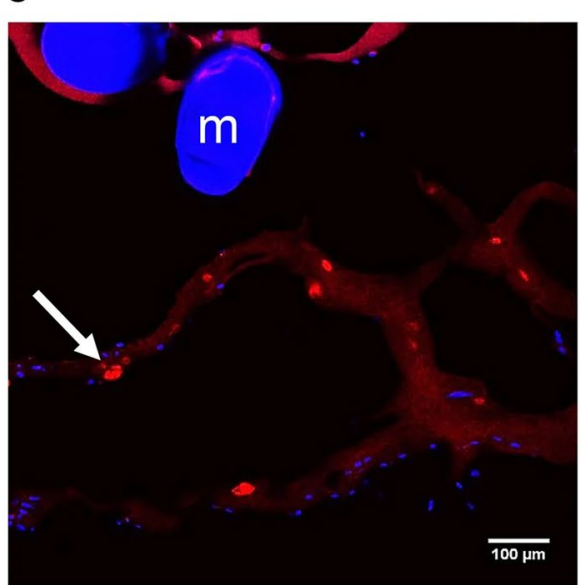

g

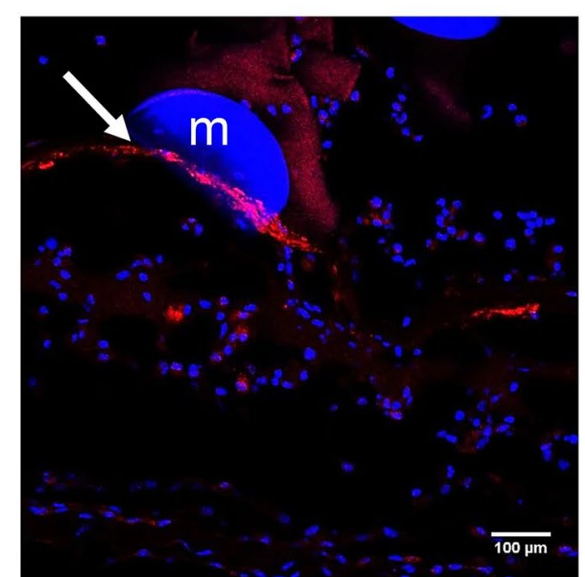

Fig. 4 eMSC transduction and survival of eMSC on PA + G mesh in NSG mice. a cultured mCherry transduced eMSC showing red fluorescence, b more than $95 \%$ of transduced and cultured eMSC were mCherry + by flow cytometry and about $40 \%$ of this population were SUSD2+. Representative trace of $n=6$ patient samples, $\mathbf{c} P A+G$ mesh seeded and cultured with eMSC. $\mathbf{d}$, e mCherry + eMSC were observed 3 and $\mathbf{f}$, g 7 days post-implantation around the mesh filaments in immunocompromised NSG mice. Arrows, representative mCherry + eMSC; $m$, mesh filament; g, gelatin. Scale Bars $100 \mu \mathrm{m}$. Reprinted with permissions from Darzi et al. [86]

Moreover, after exosomes injection, the activation, proliferation, and differentiation of SCs were promoted; the phosphorylation of ERK was enhanced; nevertheless, the myogenic effect of exosomes almost disappeared in the presence of ERK inhibitor. 


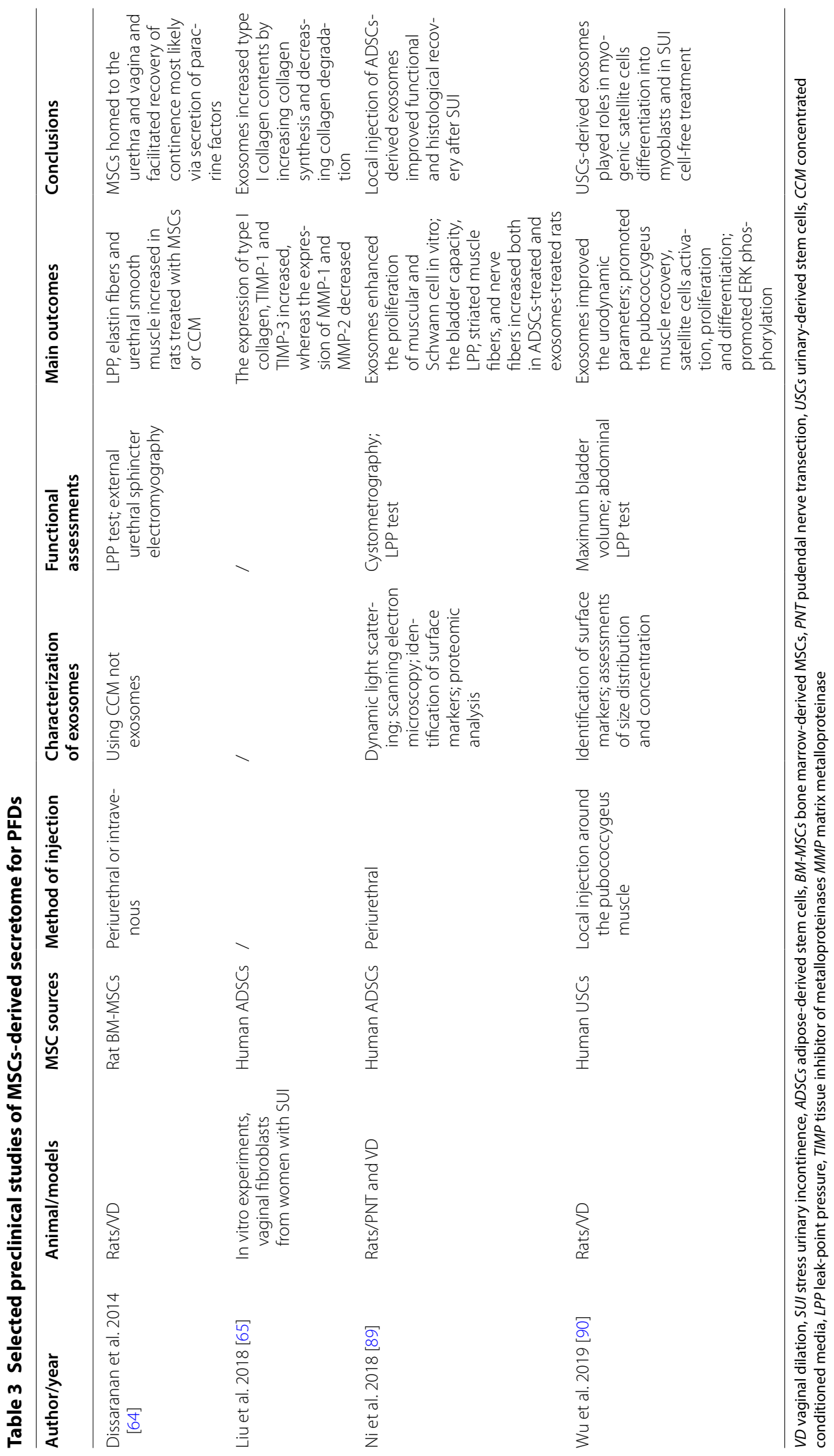


In summary, MSC-based therapies for PFDs have been tested in small animal models and have significantly improved PFDs symptoms. In these studies, rat animal models were mostly established by sphincter injury or vaginal distension and less frequently by pudendal nerve injury or vaginal incision. Such animal models are unsatisfactory because they partially mimic the disease mechanism or symptoms, and there is no gold standard animal model for PFDs. Thus, more other improved animal models should be utilized in future investigation. As for the transplanted methods, periurethral injection was used in all preclinical trials, while intravenous injection was used in some studies to classify the migration property of MSCs and to compare these two injection methods. In addition, it needs to be noticed that some of the preclinical studies reported conflicting results of the survival time and in vivo differentiation potential of the injected MSCs. These discrepancies in research outcomes may be explained by the fact that minor differences in the cell sources, culture conditions and cell dosages between these studies can profoundly affect the functions of the injected MSCs as well as the effectiveness of the therapy. Therefore, future research should focus on determining a standardized protocol of MSC-based therapy for PFDs.

\section{Clinical trials of MSCs-based therapy for PFDs}

The effectiveness of MSCs transplantation therapy in PFDs has been demonstrated in pre-clinical studies, leading to its evaluation in several clinical studies. To date, only a few small clinical studies focusing on MSCs therapy for SUI and FI have been reported. Most of them are phase I/II clinical trials, with a small number of subjects (Table 4).

Clinical trials investigating the treatment of SUI using autologous muscle derived cells (AMDCs) have shown the treatment was well-tolerated and, in some subjects, effective. Notably, AMDCs used in these trials are not a simple cell population, but a mixture of fibroblasts and myogenic cells which were identified through skeletal muscle marker expression. Sèbe et al. [91] evaluated the safety and efficacy for the intrasphincteric injections of AMDCs in women with severe SUI. There were no severe adverse effects, and three cases of urinary tract infection were reported according to the positive urine culture. Wojcikiewicz et al. [92] published a 2-year follow-up investigation on AMDCs in SUI, with a $75 \%$ success rate according to the stress test evaluations and questionnaire scores. Then the authors conducted assessments based on validated questionnaires at 2 and 4 years after the cell therapy, and the autologous cell therapy significantly improves quality of life as well as psychological condition in those patients [93]. To identify the optimal cell dose for cell therapy, Carr et al. [94] compared different intrasphincteric injection doses (varying from $1 \times 10^{6}$ to $128 \times 10^{6}$ ) of AMDCs, and better clinical outcomes were observed in patients with higher doses. Using the same method of cell isolation and urinary incontinence evaluations, Peters et al. [95] researched with an expanded sample and determined 4 different cell doses were effective and tolerated in SUI patients.

Similarly, adipose derived MSCs also have been used for the treatment of SUI in clinical trials. A pilot study conducted by Kuismanen et al. [96] revealed that 3 of 5 patients displayed a negative cough test and questionnaires showed subjective improvement in all patients at 1 year after the injection of ADSCs with collagen gel. Arjmand et al. [97] transplanted ADSCs into the periurethral region of 10 patients and presented a short-term outcome of the treatment. Urinary incontinence was significantly improved, and no complication was reported except one patient experienced slight voiding difficulty.

In addition to SUI, fecal incontinence, as another condition in PFDs, has been investigated with MSCs transplantation in clinical research. Frudinger et al. [98] injected AMDCs into the external anal sphincter in 39 (34 females and 5 males) patients and found the weekly incontinence episodes frequency reduced, with a high degree of patients' satisfaction. However, despite of the positive outcome of symptoms, the authors didn't observe physiological changes by anorectal manometry or ultrasound. Sarveazad et al. [99] conducted a randomized double-blind clinical trial to evaluate the efficacy of ADSCs transplantation in 20 female patients with sphincter defects. There was no difference of the Wexner score that was used to check muscle function between cell group and control group; the endorectal sonography and electromyography results showed an increase of muscle tissue at the repair site, but the results were not confirmed by biopsy or magnetic resonance imaging.

In 2018, a double-blind, randomized, placebo-controlled clinical trial (Fig. 5) [100] was published to evaluate the safety and efficacy of AMDCs in female subjects with SUI. The primary outcome data included stress incontinence episode frequency (IEF), 24-h in-home pad tests, in-office pad tests and Incontinence Quality of Life Scale (IQOL). The responder rates over 12 months for the composite endpoints that included at least $50 \%$ reductions in stress IEF, or 24-h pad weight test, or in-office pad weight test compared with baseline were similar between placebo and AMDCs groups, suggesting a high placebo responder rate. Then by using the more stringent endpoints that included at least $75 \%$ reduction in stress IEF or at most 1 stress incontinence episode reported over 3 days, a greater reduction of the responder rate was observed in the placebo group compared with AMDCs 


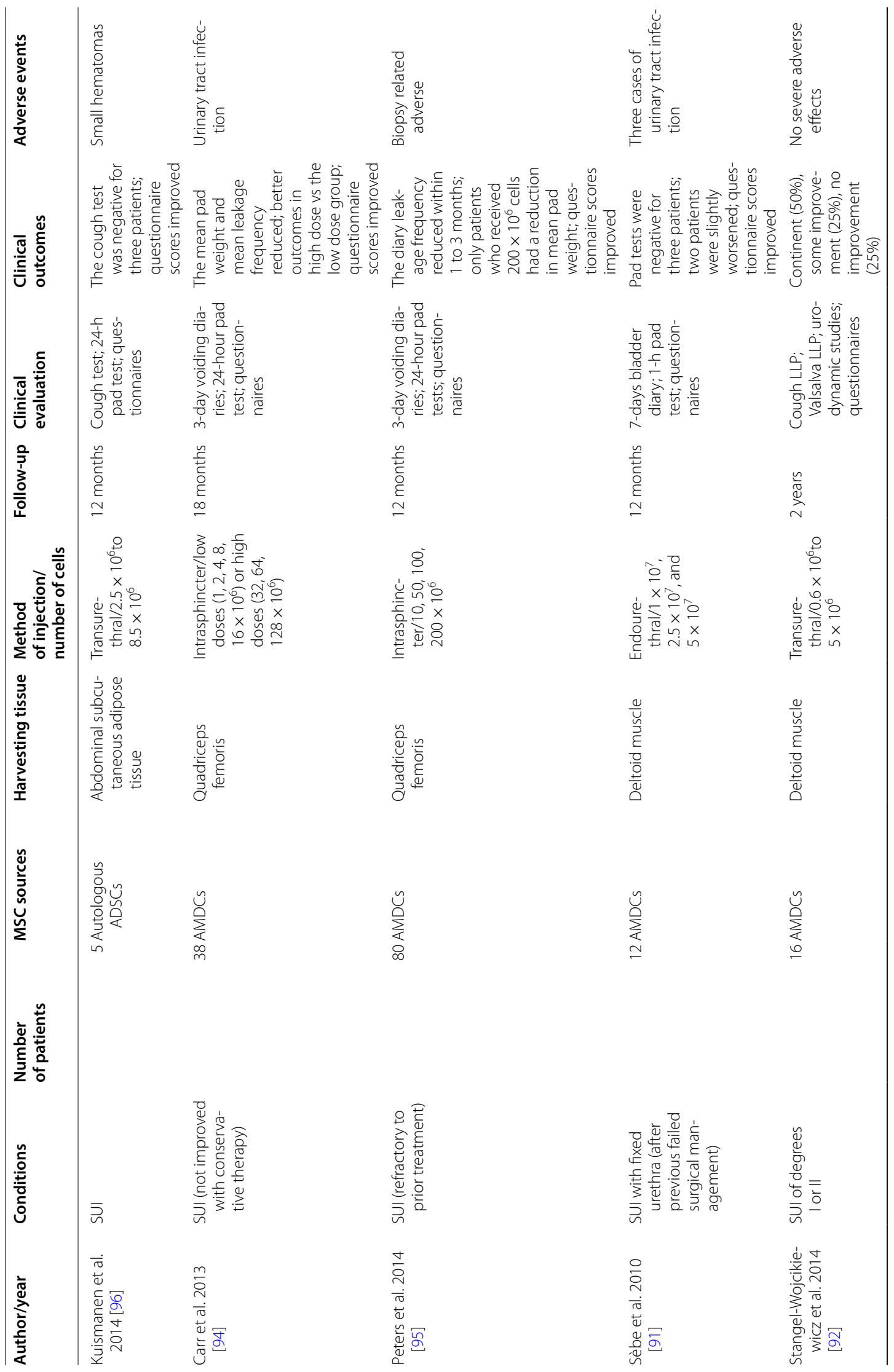


Sima and Chen Cell Biosci $\quad$ (2020) 10:104

Page 17 of 21

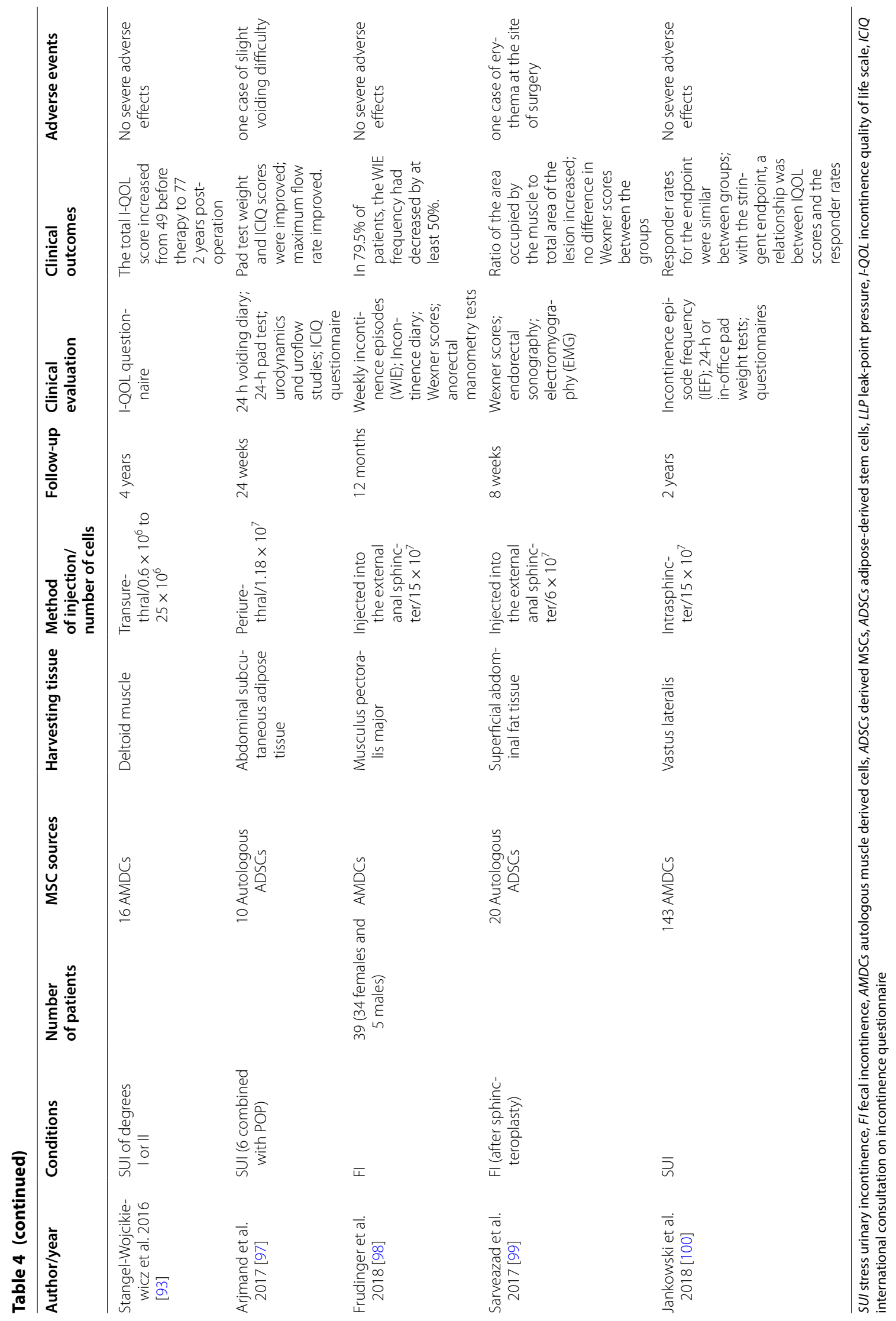




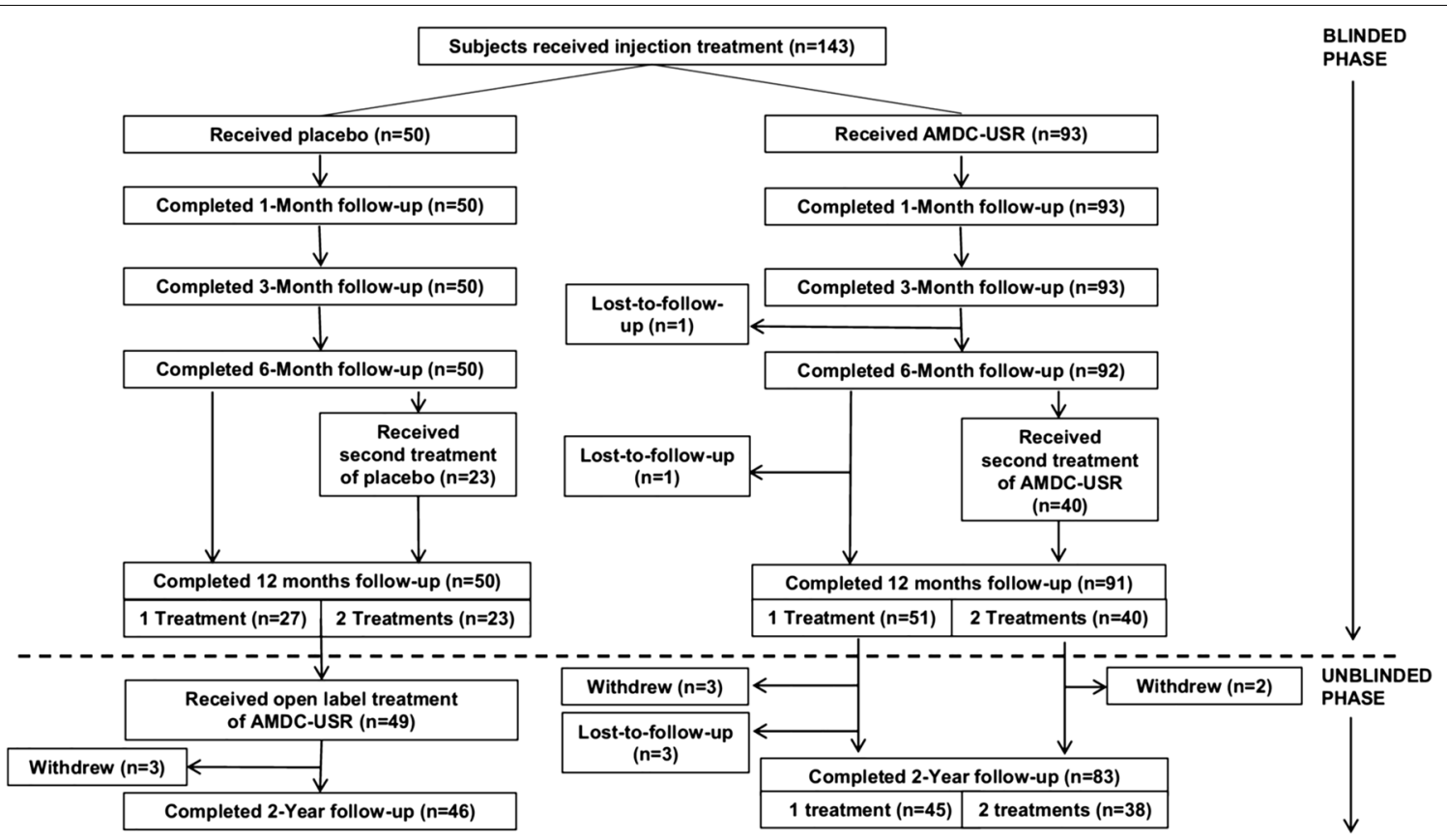

Fig. 5 Subject disposition. AMDC-USR autologous muscle derived cells for urinary sphincter repair; n number of subjects. Reprinted with permissions from Ron J. Jankowski et al. (2018)

group, but the difference was not statistically significant. Besides, the improvements in median IQOL scores were statistically significant higher for subjects who indicated a response to therapy compared with subjects who did not, according to the stringent endpoint. This post hoc analysis of the relationship between reduction in stress IEF and change in IQOL scores substantiated stress IEF as a clinically meaningful endpoint that may be used to better assess efficacy in future studies. In conclusion, although the interim analysis revealed an unexpectedly high placebo response rate and that resulted in a decrease in the evaluable sample size, the clinical trial is the largest clinical study to date in investigations on cell therapy for SUI and demonstrated AMDCs transplantation as a well-tolerated treatment for SUI. Besides, a large placebo effect is common among the clinical trials of cell therapy, making the efficacy of this therapy difficult to be evaluated in clinical trials. In spite of its limitations, the study certainly adds to our understanding of cell therapy for SUI and provides references on the study design for the future trials.

The use of MSCs seems to be a feasible and safe strategy with therapeutic effects for patients with SUI. However, as a result of the heterogeneities in preclinical and clinical trials, the standardized protocol of MSC-based therapy in SUI is still under investigation. As yet, there has been no optimal choice of cell types, cell doses, and cell injection methods for the investigation of autologous
MSCs in the therapy of PFDs. Moreover, unlike in animal studies, it is difficult to trace the injected cell fate in clinical trials. This indicates a need to find appropriate avenues for human subjects to detect the cell distribution, proliferation, and differentiation of in vivo MSCs. In addition, the therapeutic efficacy is not as good as expected in clinical trials; some patients were not responsible to the therapy. The reason for the low efficacy of cell therapy in patients with SUI is not clear but it may have something to do with the insufficient precision of cell delivery. A preclinical study [101] aiming to determine the injection accuracy rate both with transurethral and periurethral route was performed in female goats. Although majority of cell depots were administrated accurately into the urethral wall, the precise delivery of cells into external urethral sphincter is limited regardless of injection method.

In conclusion, whilst these current clinical trials did not confirm the effectiveness and safety of MSCs transplantation, they did partially substantiate MSCs transplantation as a promising alternative therapy for urinary and fecal incontinence by improving the urethral or anal sphincter function. Notwithstanding the relatively limited samples in these studies, they offer valuable insights into MSCs application in disease therapy and provide references for the future studies. Further clinical trials, with larger sample size, unified MSCs handling methods, 
and incorporation of a placebo control group, could shed more light on investigating MSC-based therapy for PFDs.

\section{Conclusion}

Currently, as the most commonly used cells in regenerative medicine, MSCs are highly investigated for PFDs owing to their rich sources, convenient acquisition, and pleiotropic effects. Existing preclinical research recognizes that MSCs exhibit a strong capacity for tissue regeneration and immune modulation by delivery of MSCs or MSCs secretions or MSCs seeded meshes. Furthermore, the therapeutic effects of MSCs transplantation for PFDs have also been underscored by several clinical trials. Whilst the mechanisms that underpin the therapeutic effects are not fully understood, in current studies, MSCs are considered as acting by secreting a large array of bioactive molecules to optimize the target cell functions and to regulate the immune responses.

These findings provide the following insights for future research. Firstly, basic work is needed to fully understand the nature of MSCs, which includes their origins, biomarkers, and biological properties. Secondly, a standardized protocol of MSC-based therapy should be established since the heterogeneous procedures for cell isolation, cultivation, and transplantation would pose a risk to the safety of MSCs clinical applications. Last but not least, future randomized controlled trials with large sample size should be carried out to evaluate the efficacy and safety of MSC-based therapy for PFDs.

Besides, as MSCs-derived extracellular vesicle such as exosome is fast becoming a key instrument in tissue repair and regeneration, acellular therapy is now regarded as a promising strategy for PFDs. Its therapeutic effects have been observed in animal models, and there's no worry about the safety issues related to cell transplantation. At present, there are few clinical trials of acellular therapy that are conducted to validate the effectiveness in patients with PFDs. Therefore, the development of acellular treatments offers a significant opportunity in the process of seeking new therapies for PFDs.

Undoubtedly, MSCs possess the therapeutic potential for PFDs as well as many other diseases, but MSC-based therapy for PFDs is still at an experimental stage. Moving forward, more investigations need to be conducted to improve the efficacy and ensure the safety of MSCsbased therapy before it is applied to the clinical treatment of PFDs.

\section{Abbreviations}

MSCs: Mesenchymal stem cells; PFDs: Pelvic floor disorders; UI: Urinary incontinence; SUI: Stress urinary incontinence; FI: Fecal incontinence; POP: Pelvic organ prolapse; GVHD: Graft-versus-host disease; BM-MSCs: Bone marrow-derived MSCs; ADSCs: Adipose-derived stem cells; eMSCs: Endometrial MSCs; MDSCs: Muscle-derived stem cells; UC-MSCs: Umbilical cordderived MSCs; HUCPVCs: Human umbilical cord perivascular cells; UCB-MSCs: Umbilical cord blood-derived MSCs; USCs: Urinary-derived stem cells; HLA-II: Human leukocyte antigen II; IL: Interleukin; TGF-b: Transforming growth factor beta; PGE: Prostaglandins E; FBR: Foreign body response; GFP: Green fluorescent protein; BLI: Bioluminescence imaging; CCM: Concentrated conditioned media; MMP: Matrix metalloproteinase; TIMP: Tissue inhibitor of metalloproteinases; PNT: Pudendal nerve transection; PNC: Pudendal nerve crush; VD: Vaginal dilation; SP: Sphincterotomy; AMDCs: Autologous muscle derived cells; ERK: Extracellular-regulated protein kinases; SCs: Satellite cells; IEF: Incontinence episode frequency; IQOL: Incontinence quality of life scale.

\section{Acknowledgements}

We would like to acknowledge my indebtedness to men of important achievements in this field, from whose lectures we benefited greatly.

\section{Authors' contributions}

YS conceived the idea and drafted the manuscript. YC revised the manuscript critically for important intellectual content, and final approval of the version to be published. All authors read and approved the final manuscript.

\section{Funding}

Not applicable.

Availability of data and materials

Not applicable.

Ethics approval and consent to participate

Not applicable.

\section{Consent for publication}

Not applicable.

\section{Competing interests}

The authors declare that they have no competing interests.

Received: 28 April 2020 Accepted: 27 August 2020

Published online: 10 September 2020

\section{References}

1. Wu JM, et al. Prevalence and trends of symptomatic pelvic floor disorders in US women. Obstet Gynecol. 2014;123(1):141-8.

2. Dooley $Y$, et al. Urinary incontinence prevalence: results from the National Health and Nutrition Examination Survey. J Urol. 2008;179(2):656-61.

3. Vergeldt TF, et al. Risk factors for pelvic organ prolapse and its recurrence: a systematic review. Int Urogynecol J. 2015;26(11):1559-73.

4. Kieserman-Shmokler C, et al. From molecular to macro: the key role of the apical ligaments in uterovaginal support. Am J Obstet Gynecol. 2020;222(5):427-36.

5. Handa VL, et al. Pelvic organ prolapse as a function of levator ani avulsion, hiatus size, and strength. Am J Obstet Gynecol. 2019;221(1):41. e1-7.

6. Madill SJ, et al. Changes in urethral sphincter size following rehabilitation in older women with stress urinary incontinence. Int Urogynecol J. 2015;26(2):277-83.

7. Arnouk A, et al. Physical, complementary, and alternative medicine in the treatment of pelvic floor disorders. Curr Urol Rep. 2017;18(6):47.

8. Morling JR, et al. Adverse events after first, single, mesh and non-mesh surgical procedures for stress urinary incontinence and pelvic organ prolapse in Scotland, 1997-2016: a population-based cohort study. Lancet. 2017;389(10069):629-40.

9. Glazener CM, et al. Mesh, graft, or standard repair for women having primary transvaginal anterior or posterior compartment prolapse surgery: two parallel-group, multicentre, randomised, controlled trials (PROSPECT). Lancet. 2017;389(10067):381-92. 
10. Le Blanc K, et al. Mesenchymal stem cells for treatment of steroid-resistant, severe, acute graft-versus-host disease: a phase II study. Lancet. 2008:371(9624):1579-86.

11. Hare JM, et al. Comparison of allogeneic vs autologous bone marrowderived mesenchymal stem cells delivered by transendocardial injection in patients with ischemic cardiomyopathy: the POSEIDON randomized trial. JAMA. 2012:308(22):2369-79.

12. Karussis $D$, et al. Safety and immunological effects of mesenchymal stem cell transplantation in patients with multiple sclerosis and amyotrophic lateral sclerosis. Arch Neurol. 2010;67(10):1187-94.

13. Friedenstein AJ, et al. Precursors for fibroblasts in different populations of hematopoietic cells as detected by the in vitro colony assay method. Exp Hematol. 1974;2(2):83-92.

14. Pittenger MF, et al. Multilineage potential of adult human mesenchymal stem cells. Science. 1999;284(5411):143-7.

15. Zuk PA, et al. Human adipose tissue is a source of multipotent stem cells. Mol Biol Cell. 2002;13(12):4279-95.

16. Ding DC, et al. Human umbilical cord mesenchymal stem cells: a new era for stem cell therapy. Cell Transplant. 2015;24(3):339-47.

17. Anker PS, et al. Isolation of mesenchymal stem cells of fetal or maternal origin from human placenta. Stem cells. 2004;22(7):1338-45.

18. Alviano F, et al. Term Amniotic membrane is a high throughput source for multipotent Mesenchymal Stem Cells with the ability to differentiate into endothelial cells in vitro. BMC Dev Biol. 2007;7:11.

19. Toma JG, et al. Isolation and characterization of multipotent skinderived precursors from human skin. Stem Cells. 2005;23(6):727-37.

20. Gronthos S, et al. Postnatal human dental pulp stem cells (DPSCs) in vitro and in vivo. Proc Natl Acad Sci U S A. 2000;97(25):13625-30.

21. Dominici $M$, et al. Minimal criteria for defining multipotent mesenchymal stromal cells. The International Society for Cellular Therapy position statement. Cytotherapy. 2006:8(4):315-7.

22. Bartunek J, et al. Congestive Heart Failure Cardiopoietic Regenerative Therapy (CHART-1) trial design. Eur J Heart Fail. 2016;18(2):160-8.

23. Panes J, et al. Expanded allogeneic adipose-derived mesenchymal stem cells (Cx601) for complex perianal fistulas in Crohn's disease: a phase 3 randomised, double-blind controlled trial. Lancet. 2016:388(10051):1281-90.

24. Galipeau J, Sensebe L. Mesenchymal Stromal Cells: clinical Challenges and Therapeutic Opportunities. Cell Stem Cell. 2018;22(6):824-33.

25. Lv FJ, et al. Concise review: the surface markers and identity of human mesenchymal stem cells. Stem Cells. 2014;32(6):1408-19.

26. Kwon A, et al. Tissue-specific differentiation potency of mesenchymal stromal cells from perinatal tissues. Sci Rep. 2016;6:23544.

27. Billing AM, et al. Comprehensive transcriptomic and proteomic characterization of human mesenchymal stem cells reveals source specific cellular markers. Sci Rep. 2016;6:21507.

28. Crisan $\mathrm{M}$, et al. A perivascular origin for mesenchymal stem cells in multiple human organs. Cell Stem Cell. 2008;3(3):301-13.

29. Sacchetti B, et al. No identical "Mesenchymal Stem Cells" at different times and sites: human committed progenitors of distinct origin and differentiation potential are incorporated as adventitial cells in microvessels. Stem Cell Reports. 2016;6(6):897-913.

30. Ranganath $\mathrm{SH}$, et al. Harnessing the mesenchymal stem cell secretome for the treatment of cardiovascular disease. Cell Stem Cell. 2012;10(3):244-58.

31. Cruz FF, et al. Systemic administration of human bone marrow-derived mesenchymal stromal cell extracellular vesicles ameliorates aspergillus hyphal extract-induced allergic airway inflammation in immunocompetent mice. Stem Cells Transl Med. 2015;4(11):1302-16.

32. Lener T, et al. Applying extracellular vesicles based therapeutics in clinical trials_an ISEV position paper. J Extracell Vesicles. 2015;4:30087.

33. Caplan Al. Mesenchymal stem cells: time to change the name! Stem Cells Transl Med. 2017;6(6):1445-51.

34. Sipp D, Robey PG, Turner L. Clear up this stem-cell mess. Nature. 2018;561(7724):455-7.

35. Kozlowska U, et al. Similarities and differences between mesenchymal stem/progenitor cells derived from various human tissues. World J Stem Cells. 2019;11(6):347-74.

36. Heo JS, et al. Comparison of molecular profiles of human mesenchymal stem cells derived from bone marrow, umbilical cord blood, placenta and adipose tissue. Int J Mol Med. 2016;37(1):115-25.
37. Muller I, et al. Application of multipotent mesenchymal stromal cells in pediatric patients following allogeneic stem cell transplantation. Blood Cells Mol Dis. 2008;40(1):25-32.

38. Lin $\mathrm{G}$, et al. Treatment of stress urinary incontinence with adipose tissue-derived stem cells. Cytotherapy. 2010;12(1):88-95.

39. Gotoh M, et al. Regenerative treatment of male stress urinary incontinence by periurethral injection of autologous adiposederived regenerative cells: 1-year outcomes in 11 patients. Int J Urol. 2014;21(3):294-300.

40. Klimczak A, Kozlowska U, Kurpisz M. Muscle stem/progenitor cells and mesenchymal stem cells of bone marrow origin for skeletal muscle regeneration in muscular dystrophies. Arch Immunol Ther Exp (Warsz). 2018;66(5):341-54.

41. Pannerec A, et al. Defining skeletal muscle resident progenitors and their cell fate potentials. Development. 2013;140(14):2879-91.

42. Ho MH, et al. Stimulating vaginal repair in rats through skeletal muscle-derived stem cells seeded on small intestinal submucosal scaffolds. Obstet Gynecol. 2009:114(2):300-9.

43. Gargett CE, et al. Isolation and culture of epithelial progenitors and mesenchymal stem cells from human endometrium. Biol Reprod. 2009;80(6):1136-45.

44. Dimitrov $\mathrm{R}$, et al. Characterization of clonogenic stromal cells isolated from human endometrium. Reproduction. 2008;135(4):551-8.

45. Mukherjee $\mathrm{S}$, et al. Blended nanostructured degradable mesh with endometrial mesenchymal stem cells promotes tissue integration and anti-inflammatory response in vivo for pelvic floor application. Biomacromol. 2019;20(1):454-68.

46. Paul K, et al. 3D bioprinted endometrial stem cells on melt electrospun poly epsilon-caprolactone mesh for pelvic floor application promote anti-inflammatory responses in mice. Acta Biomater. 2019;97:162-76.

47. Ulrich D, et al. Mesenchymal stem/stromal cells in post-menopausal endometrium. Hum Reprod. 2014;29(9):1895-905.

48. Baksh D, Yao R, Tuan RS. Comparison of proliferative and multilineage differentiation potential of human mesenchymal stem cells derived from umbilical cord and bone marrow. Stem Cells. 2007;25(6):1384-92.

49. Ding J, et al. Induction of human umbilical cord mesenchymal stem cells into tissue-forming cells in a murine model: implications for pelvic floor reconstruction. Cell Tissue Res. 2018;372(3):535-47.

50. Lee CN, et al. Human cord blood stem cell therapy for treatment of stress urinary incontinence. J Korean Med Sci. 2010;25(6):813-6.

51. Macias Ml, et al. Isolation and characterization of true mesenchymal stem cells derived from human term decidua capable of multilineage differentiation into all 3 embryonic layers. Am J Obstet Gynecol. 2010;203(5):495.e9-23.

52. Bharadwaj S, et al. Multipotential differentiation of human urinederived stem cells: potential for therapeutic applications in urology. Stem Cells. 2013;31(9):1840-56.

53. Zhang $\mathrm{Y}$, et al. Urine derived cells are a potential source for urological tissue reconstruction. J Urol. 2008;180(5):2226-33.

54. Karp JM, Leng Teo GS. Mesenchymal stem cell homing: the devil is in the details. Cell Stem Cell. 2009:4(3):206-16.

55. Wynn RF, et al. A small proportion of mesenchymal stem cells strongly expresses functionally active CXCR4 receptor capable of promoting migration to bone marrow. Blood. 2004;104(9):2643-5.

56. De Ugarte DA, et al. Differential expression of stem cell mobilizationassociated molecules on multi-lineage cells from adipose tissue and bone marrow. Immunol Lett. 2003;89(2-3):267-70.

57. Cruz M, et al. Pelvic organ distribution of mesenchymal stem cells injected intravenously after simulated childbirth injury in female rats. Obstet Gynecol Int. 2012;2012:612946.

58. Ben Menachem-Zidon O, et al. Systemically transplanted mesenchymal stem cells induce vascular-like structure formation in a rat model of vaginal injury. PLoS ONE. 2019;14(6):e0218081.

59. von Bahr $\mathrm{L}$, et al. Analysis of tissues following mesenchymal stromal cell therapy in humans indicates limited long-term engraftment and no ectopic tissue formation. Stem Cells. 2012;30(7):1575-8.

60. Gill BC, Sun DZ, Damaser MS. Stem cells for urinary incontinence: functional differentiation or cytokine effects? Urology. 2018;117:9-17. 
61. Keshtkar S, Azarpira N, Ghahremani MH. Mesenchymal stem cellderived extracellular vesicles: novel frontiers in regenerative medicine. Stem Cell Res Ther. 2018;9(1):63.

62. Phinney DG, Pittenger MF. Concise review: mSC-Derived exosomes for cell-free therapy. Stem Cells. 2017;35(4):851-8.

63. Spees JL, Lee RH, Gregory CA. Mechanisms of mesenchymal stem/ stromal cell function. Stem Cell Res Ther. 2016;7(1):125.

64. Dissaranan C, et al. Rat mesenchymal stem cell secretome promotes elastogenesis and facilitates recovery from simulated childbirth injury. Cell Transplant. 2014;23(11):1395-406.

65. Liu $X$, et al. Exosomes secreted by adipose-derived mesenchymal stem cells regulate type I collagen metabolism in fibroblasts from women with stress urinary incontinence. Stem Cell Res Ther. 2018;9(1):159.

66. $\mathrm{Hu} L$, et al. Exosomes derived from human adipose mensenchymal stem cells accelerates cutaneous wound healing via optimizing the characteristics of fibroblasts. Sci Rep. 2016;6:32993.

67. Huuskes BM, et al. Combination therapy of mesenchymal stem cells and serelaxin effectively attenuates renal fibrosis in obstructive nephropathy. Faseb j. 2015;29(2):540-53.

68. El Agha E, et al. Mesenchymal Stem Cells in Fibrotic Disease. Cell Stem Cell. 2017;21(2):166-77.

69. Le Blanc K, et al. HLA expression and immunologic properties of differentiated and undifferentiated mesenchymal stem cells. Exp Hematol. 2003;31(10):890-6.

70. Tse WT, et al. Suppression of allogeneic T-cell proliferation by human marrow stromal cells: implications in transplantation. Transplantation. 2003;75(3):389-97.

71. Ankrum JA, Ong JF, Karp JM. Mesenchymal stem cells: immune evasive, not immune privileged. Nat Biotechnol. 2014;32(3):252-60.

72. Le Blanc K, Mougiakakos D. Multipotent mesenchymal stromal cells and the innate immune system. Nat Rev Immunol. 2012;12(5):383-96.

73. Li N, Hua J. Interactions between mesenchymal stem cells and the immune system. Cell Mol Life Sci. 2017;74(13):2345-60.

74. Gupta N, et al. Intrapulmonary delivery of bone marrow-derived mesenchymal stem cells improves survival and attenuates endotoxininduced acute lung injury in mice. J Immunol. 2007;179(3):1855-63.

75. Nemeth $\mathrm{K}$, et al. Bone marrow stromal cells attenuate sepsis via prostaglandin $\mathrm{E}(2)$-dependent reprogramming of host macrophages to increase their interleukin-10 production. Nat Med. 2009;15(1):42-9.

76. Miao CM, et al. Bone marrow stromal cells attenuate LPS-induced mouse acute liver injury via the prostaglandin E 2-dependent repression of the NLRP3 inflammasome in Kupffer cells. Immunol Lett. 2016;179:102-13.

77. Anderson JM, Rodriguez A, Chang DT. Foreign body reaction to biomaterials. Semin Immunol. 2008;20(2):86-100.

78. Ulrich $D$, et al. Human endometrial mesenchymal stem cells modulate the tissue response and mechanical behavior of polyamide mesh implants for pelvic organ prolapse repair. Tissue Eng Part A. 2014;20(3-4):785-98.

79. Mukherjee $S$, et al. Mesenchymal stem cell-based bioengineered constructs: foreign body response, cross-talk with macrophages and impact of biomaterial design strategies for pelvic floor disorders. Interface Focus. 2019;9(4):20180089.

80. Sadeghi Z, et al. Mesenchymal stem cell therapy in a rat model of birth-trauma injury: functional improvements and biodistribution. Int Urogynecol J. 2016;27(2):291-300.

81. Salcedo $L$, et al. Mesenchymal stem cells can improve anal pressures after anal sphincter injury. Stem Cell Res. 2013;10(1):95-102.

82. Salcedo $L$, et al. Functional outcome after anal sphincter injury and treatment with mesenchymal stem cells. Stem Cells Transl Med. 2014;3(6):760-7.

83. Kuismanen $\mathrm{K}$, et al. Functional outcome of human adipose stem cel injections in rat anal sphincter acute injury model. Stem Cells Transl Med. 2018;7(3):295-304

84. Williams JK, et al. The dose-effect safety profile of skeletal muscle precursor cell therapy in a dog model of intrinsic urinary sphincter deficiency. Stem Cells Transl Med. 2015;4(3):286-94.
85. Edwards SL, et al. Temporal changes in the biomechanical properties of endometrial mesenchymal stem cell seeded scaffolds in a rat model. Acta Biomater. 2015:13:286-94.

86. Darzi S, et al. Endometrial mesenchymal stem/stromal cells modulate the macrophage response to implanted polyamide/gelatin composite mesh in immunocompromised and immunocompetent mice. Sci Rep. 2018:8(1):6554

87. Sun DZ, et al. Harnessing the mesenchymal stem cell secretome for regenerative urology. Nat Rev Urol. 2019;16(6):363-75.

88. Deng K, et al. Mesenchymal stem cells and their secretome partially restore nerve and urethral function in a dual muscle and nerve injury stress urinary incontinence model. Am J Physiol Renal Physiol. 2015;308(2):F92-f100.

89. $\mathrm{Ni}$ J, et al. Therapeutic potential of human adipose-derived stem cell exosomes in stress urinary incontinence-an in vitro and in vivo study. Cell Physiol Biochem. 2018;48(4):1710-22.

90. Wu R, et al. Exosomes secreted by urine-derived stem cells improve stress urinary incontinence by promoting repair of pubococcygeus muscle injury in rats. Stem Cell Res Ther. 2019;10(1):80.

91. Sebe P, et al. Intrasphincteric injections of autologous muscular cells in women with refractory stress urinary incontinence: a prospective study. Int Urogynecol J. 2011;22(2):183-9.

92. Stangel-Wojcikiewicz K, et al. Autologous muscle-derived cells for the treatment of female stress urinary incontinence: a 2-year follow-up of a Polish investigation. Neurourol Urodyn. 2014;33(3):324-30.

93. Stangel-Wojcikiewicz K, et al. Quality of life assessment in female patients 2 and 4 years after muscle-derived cell transplants for stress urinary incontinence treatment. Ginekol Pol. 2016;87(3):183-9.

94. Carr LK, et al. Autologous muscle derived cell therapy for stress urinary incontinence: a prospective, dose ranging study. J Urol. 2013;189(2):595-601.

95. Peters KM, et al. Autologous muscle derived cells for treatment of stress urinary incontinence in women. J Urol. 2014;192(2):469-76.

96. Kuismanen $\mathrm{K}$, et al. Autologous adipose stem cells in treatment of female stress urinary incontinence: results of a pilot study. Stem Cells Transl Med. 2014;3(8):936-41.

97. Arjmand B, et al. Concomitant transurethral and transvaginal-periurethral injection of autologous adipose derived stem cells for treatment of female stress urinary incontinence: a phase one clinical trial. Acta Med Iran. 2017;55(6):368-74.

98. Frudinger A, et al. Skeletal muscle-derived cell implantation for the treatment of sphincter-related faecal incontinence. Stem Cell Res Ther. 2018;9(1):233.

99. Sarveazad A, et al. A new method for treating fecal incontinence by implanting stem cells derived from human adipose tissue: preliminary findings of a randomized double-blind clinical trial. Stem Cell Res Ther. 2017:8(1):40.

100. Jankowski RJ, et al. A double-blind, randomized, placebo-controlled clinical trial evaluating the safety and efficacy of autologous muscle derived cells in female subjects with stress urinary incontinence. Int Urol Nephrol. 2018;50(12):2153-65.

101. Burdzinska A, et al. Limited accuracy of transurethral and periurethral intrasphincteric injections of cellular suspension. Neurourol Urodyn. 2018;37(5):1612-22

102. Silwal Gautam S, et al. Implantation of autologous adipose-derived cells reconstructs functional urethral sphincters in rabbit cryoinjured urethra. Tissue Eng Part A. 2014;20(13-14):1971-9.

\section{Publisher's Note}

Springer Nature remains neutral with regard to jurisdictional claims in published maps and institutional affiliations. 